\title{
Distribution-Free CUSUM-Type Control Charts for Monitoring Industrial Processes: An Overview
}

\author{
Ioannis S. Triantafyllou \\ Department of Computer Science \& Biomedical Informatics, \\ University of Thessaly, Lamia 35131, Greece. \\ Corresponding author: itriantafyllou@uth.gr \\ Mangey Ram \\ Department of Mathematics, Computer Science \& Engineering, \\ Graphic Era Deemed to be University, Dehradun-248002, Uttarakhand, India. \\ E-mail: mangeyram@gmail.com
}

(Received on January 23, 2021; Accepted on April 3, 2021)

\begin{abstract}
In the present paper we provide an up-to-date overview of nonparametric Cumulative Sum (CUSUM) monitoring schemes. Due to their nonparametric nature, such memory-type schemes are proved to be very useful for monitoring industrial processes, where the output does not match to a particular distributional model. Several fundamental contributions on the topic are mentioned, while recent advances are also presented in some detail. In addition, some practical applications of the nonparametric CUSUM-type control charts are highlighted, in order to emphasize their crucial role in the contemporary online Statistical Process Control.
\end{abstract}

Keywords- Distribution-free statistical methods, Rank-based procedures, Nonparametric statistical process control, Cumulative sum control charts, Sign statistics.

\section{Introduction}

Statistical Process Control is implemented in industrial procedures and aims at improving their quality by monitoring the corresponding production process. Among others, control charts are proved to be very useful statistical tools for supervising a quality characteristic of the product and tracking down plausible changes in the underlying distributional model. The most popular and easy-to-use monitoring schemes, which have been introduced and well-studied in the literature, are the so-called Shewhart-type, Cumulative Sum (CUSUM, hereafter) and Exponentially Weighted Moving Average (EWMA, hereafter) control charts. For a thorough consideration of the basic principles of the topic, the excellent monographs of Montgomery (2007), Qiu (2014) and the works of Woodall and Montgomery (1999), Woodall (2000) are strongly recommended.

A broad variety of monitoring schemes have been already established under the presumption that the output matches to a particular distributional model. Nevertheless, this demand is not constantly fulfilled and consequently it is evident that the distribution-based monitoring schemes do not constantly provide a reliable framework. To overcome this problem and at the same time hold the ordinary frame of a control chart, a lot of nonparametric charts are already known. These charts are independent from the process distribution and therefore any distributional assumptions are required. The general framework of the so-called Nonparametric Statistical Process Control and some fundamental perspectives of it could be found in the works of Qiu and Li (2011a, 2011b), Chakraborti and Graham (2019a), Qiu (2018). 
One of the classes of distribution-free schemes for monitoring univariate processes, is the family of the Shewhart-type nonparametric monitoring schemes (SNCC class, hereafter). The charts, which are included in the $S N C C$ class, utilize an appropriate nonparametric test statistic, whose values are calculated by the aid of individual observations or random groups of observations collected from the process. These observed values of the test statistic are plotted and compared to the control limits of the corresponding chart, in order to draw a conclusion about the status of the underlying process. The $S N C C$ class seems to include the easiest-to-use control charts compared to other families of distribution-free schemes. Some pioneer works on the SNCC class are presented by the review paper of Chakraborti et al. (2001), while recent advances on the topic are discussed by Chakraborti and Graham (2019b), Koutras and Triantafyllou (2020).

A second family of distribution-free monitoring schemes contains memory-type statistical tools, known as nonparametric EWMA control charts (EWMANCC class, hereafter). These charts utilize an appropriate nonparametric testing procedure in order to build the corresponding $E W M A$ statistic, whose values are afterwards plotted and monitored by the aid of the respective control limits. It is evident that the EWMANCC class is supposed to be superior to the aforementioned SNCC class for tracking down slight changes of the underlying process. Some recent advances on the topic are provided by You and Qiu (2020), Perdikis et al. (2021).

The third family of distribution-free schemes for monitoring univariate processes includes the socalled $C U S U M$ nonparametric control charts ( $C N C C$ class, hereafter). Each member of this class calls for an appropriate nonparametric test statistic and afterwards the CUSUM procedure is constructed via the conventional approach. The $C N C C$ class outperforms the $S N C C$ family for tracking down small or moderate changes of either the location or the scale parameter of the underlying process. For multivariate CUSUM nonparametric control charts, Qiu and Hawkins (2001), Qiu (2008), Xue and Qiu (2020) provided some excellent works. Throughout the lines of the present review article, the $C N C C$ class constitutes the target group of distribution-free monitoring schemes in the univariate case. The CUSUM nonparametric control charts aim at monitoring a non-normal process or even a process with an unknown distribution. In other words, the members of $C N C C$ class seem quite capable to monitor adequately a quantitative characteristic of a product, even if its distribution is heavy-tailed or skewed or it cannot be derived by historical data. The above-mentioned attribute of the CUSUM nonparametric schemes accompanied with their simplicity, makes them quite attractive for the practitioners.

The rest of the paper is formulated as follows: Section 3 offers a review of univariate nonparametric CUSUM-type control charts based on sign statistics. Such works start to appear in the literature forty years ago, while some relative outcomes have been recently published. In Section 4, we study control charts, which belong to the $C N C C$ class and are based on order statistics, while Section 5 offers a detailed presentation of $C U S U M$-type rank-based monitoring schemes. Section 6 puts forth the general outline of the present manuscript, whilst several thoughts for extending the research on the topic are also discussed.

\section{General Notations and CUSUM Framework}

In the present section, general notations are presented in order to make the remaining manuscript more readable. The main symbols which are utilized in the next sections, are listed below. However, some specific notations which are not generally used by all charts, are properly introduced and defined throughout the lines of the corresponding subsection of the present review article. 
ARL: Average Run Length

AEQL: Average Extra Quadratic Loss.

FAR: False Alarm Rate

IC: in-control

OOC: out-of-control

sign (a): the sign of number a

SR: Wilcoxon signed-rank statistic

I(b): an indicator (dummy) variable depending on $b$

LCL: Lower Control Limit

W: Wilcoxon rank-sum statistic

MW: Mann-Whitney test statistic

UCL: Upper Control Limit

RSS: Ranked set sampling

Generally speaking, the CUSUM control charts provide an attractive alternative monitoring tool, when small location or scale shifts are investigated. In fact, a CUSUM scheme exploits directly the total amount of information drawn from the sampling procedure by depicting the cumulative sums of the deviations of the sample values from the target one $(\mu)$. Due to the fact that CUSUM charts combine information from different samples, they are proved to be more capable than Shewharttype charts for tracking down slight process changes. If a shift in the process is under way, the CUSUM chart is expected to produce a signal, while an adjustment should take place in order to reinstate the process back on the desirable performance level. The tabular CUSUM is constructed by accumulating derivations from target value that are either above it (with the so-called one-sided upper CUSUM statistic $C_{1}$ ) or below the target value (with the corresponding one-sided lower CUSUM statistic $C_{2}$ ). It goes without saying that whenever either the upper or lower CUSUM statistic exceeds the decision interval, the process is characterized as OOC. A graphical representation of the general framework for constructing a CUSUM chart follows the ordinary scheme, namely the cumulative sum statistic is plotted versus the identification number. Afterwards, the process is characterized as IC or OOC by comparing its observed values to the corresponding control limits Montgomery (2007).

\section{Distribution-Free CUSUM Control Charts Based on Sign Statistics}

Throughout the lines of the present part, univariate nonparametric schemes, which rely on the wellknown sign statistic and follow the general CUSUM framework are discussed. It is evident that the sign statistic and its modifications have been already utilized for constructing Shewhart-type nonparametric schemes (Asghari et al., 2018; Castagliola et al., 2020). However, our study aims at discussing the corresponding CUSUM-type advances. The first attempt towards this direction, which has been recorded in the literature, belongs to McGilchrist and Woodyer (1975). In their article, a CUSUM technique which endures distribution-free testing is introduced and implemented for detecting possible shifts in the median of a rainfall distribution.

We next provide a detailed review of nonparametric univariate monitoring schemes based on sign statistics, namely of these members of the $C N C C$ class which employ the well-known sign statistic or its modifications. The set-up of presentation of the included articles is determined to be chronological for the purpose of setting up a comprehensible and well-structured flow. 


\subsection{A Nonparametric CUSUM Control Chart Based on Wilcoxon Signed-Rank Statistic}

Bakir and Reynolds (1979) proposed a distribution-free methodology for tracking down possible changes in the mean of a sequence of observations from a specified control value. In the framework of their distribution-free scheme, the Wilcoxon signed-rank statistic is monitored, while ranking takes place within groups. Bakir and Reynolds (1979) treated the cumulative sum procedure as a Markov chain and consequently they proposed a simple technique for determining the exact Average Run Length of the corresponding monitoring scheme. It is true that the method introduced by Bakir and Reynolds (1979) can be applied for any process distribution, under the assumption that we have at hand the distribution of the Wilcoxon signed-rank statistic for that case. Note that ranking was accomplished within groups, where the groups were either samples taken at each point or else artificially defined groups and that makes the determination of the corresponding ranks much easier. It goes without saying that if single observations are collected randomly from the production, then the observation must be artificially divided into groups.

Let us denote by $X_{i 1}, X_{i 2}, \ldots, X_{i n}, i=1,2, \ldots$ a random sample of size $n$ consisting of observations which are drawn sequentially from the process. If $R_{i j}, j=1,2, \ldots, n$ corresponds to the rank of the absolute value of $X_{i j}$, namely to the rank of $\left|X_{i j}\right|$ inside the group of observations $\left|X_{i 1}\right|,\left|X_{i 2}\right|, \ldots,\left|X_{i n}\right|, i=1,2, \ldots$ and $\operatorname{sign}(a)$ denotes the sign of number $a$, then we define the respective Wilcoxon signed-ranks within the $i$-th group as

$U_{i j}=\operatorname{sign}\left(X_{i j}\right) R_{i j}, j=1,2, \ldots, n$

The so-called Wilcoxon signed-rank statistic for the $i$-th sample can be now expressed as

$S R_{i}=\sum_{j=1}^{n} U_{i j}, i=1,2, \ldots$

Bakir and Reynolds (1979) proposed a grouped signed-rank test, which relies on the observed $S R_{i}, i=1,2, \ldots$ of the nonparametric statistic defined in (2) and simultaneously exploits the Cumulative Sum Control Chart (CSCC, hereafter) proposed by Page (1954). The CSCC aims at detecting possible shifts of the underlying distribution process either in the positive or in the negative direction. In other words, the so-called CSCC produces a signal that a positive shift has been detected at the first $d$ observed values if the following ensues.

$\sum_{i=1}^{d}\left(X_{i j}-w_{1}\right)-\min _{0 \leq m \leq d} \sum_{i=1}^{m}\left(X_{i j}-w_{1}\right) \geq h_{1}, j=1,2, \ldots, n$,

where $w_{1}$ and $h_{1}$ are design parameters of the CSCC procedure. On the other hand, the CSCC produces a signal that a negative shift has been detected at the first $d$ observed values if the following ensues. 
$\max _{0 \leq m \leq d} \sum_{i=1}^{m}\left(X_{i j}+w_{2}\right)-\sum_{i=1}^{d}\left(X_{i j}+w_{2}\right) \geq h_{2}, j=1,2, \ldots, n$

where $w_{2}$ and $h_{2}$ are design parameters of the CSCC procedure. It is straightforward that a twosided CSCC can be constructed by combining the signaling rules (3) and (4), while if we set $w_{1}=w_{2}$, $h_{1}=h_{2}$, a symmetric control chart is generated.

The proposed distribution-free CUSUM monitoring scheme can be viewed as either one- or twosided chart. More precisely, the one-sided nonparametric CUSUM control chart introduced by Bakir and Reynolds (1979) produces an alarm for a positive change in the process mean has been occurred at the first $d$ observed values if the following holds true.

$$
\sum_{i=1}^{d}\left(S R_{i}-w\right)-\min _{0 \leq m \leq d} \sum_{i=1}^{m}\left(S R_{i}-w\right) \geq h
$$

Following a similar argumentation, the one-sided nonparametric CUSUM control chart introduced by Bakir and Reynolds (1979) produces an alarm for a negative shift in the process mean has been occurred at the first $d$ observed values if

$$
\max _{0 \leq m \leq d} \sum_{i=1}^{m}\left(S R_{i}+w\right)-\sum_{i=1}^{d}\left(S R_{i}+w\right) \geq h
$$

For constructing a two-sided CUSUM chart, we should combine the signaling rules stated in Eq. (5) and (6). Note that design parameters $w$ and $h$ are determined appropriately so that the OOC behavior of the resulting monitoring scheme is optimized. In more practical terms, the monitoring scheme established by Bakir and Reynolds (1979) can be viewed as sequential testing procedure, where the cumulative sum $\sum_{i=1}^{d}\left(S R_{i}-w\right)$ for each test is accumulated till the sum either exceeds or equals to the value $h$ or is no greater than zero. In case of recording a non-positive sum at the end of a test, a new one is activated based on the next observation. The testing procedure gives an OOC alarm whenever the sum at the end of a test is equal to or exceeds the value $h$.

Under the assumption that parameter $w$ is non-negative integer, it is evident that the Wilcoxon signed-rank statistic for the $i$-th sample is also an integer inside the interval $(-n(n+1) / 2, n(n+1) / 2)$. Therefore, the quantity $\sum_{i=1}^{d}\left(S R_{i}-w\right)$ shall be also integer valued. Bakir and Reynolds (1979) stated that the time their CUSUM testing procedure signals coincides to the first time point that the discrete Markov chain $\left\{S_{v}: v=0,1, \ldots\right\}$ enters the state $h$, where the state space is given as $\{0,1, \ldots, h\}, S_{0}=0$ and $S_{n}=\min \left\{h, \max \left\{0, S_{n-1}+S R_{n}-w\right\}\right\}$. It is noticeable that if state $h$ is viewed as an absorbing state, then the expected amount of groups of observations for the procedure is equivalent to the average time to absorption into state $h$. Based on the last argument, Bakir and Reynolds (1979) expressed the ARL of their CUSUM scheme as 
$n m_{0}$, where $m_{0}$ corresponds to the mean time to absorption under the presumption that the chain started initially at state 0 .

From the comparisons accomplished by Bakir and Reynolds (1979), it is concluded that in case where the observations are naturally drawn in groups, their CUSUM monitoring scheme performs slightly worse compared to the competitive parametric ones under the normality presumption, while it is proved to be considerably more capable than the parametric techniques for non-normal distributions.

\subsection{A Nonparametric CUSUM Control Chart Based on the Sign Test Statistic}

Amin et al. (1995) proposed both one-sided and two-sided CUSUM charts which rely on the sign test statistic. It is true that $\mathrm{n}$ the case of monitoring schemes based on signs, the corresponding Average Run Length remains stable for all continuous distributional models, given that the respective median is equal to the target value. The nonparametric monitoring schemes introduced by Amin et al. (1995) aim at supervising the process median (or mean) and rely on signs computed within samples. More specifically, the proposed CUSUM control chart utilizes a cumulative sum of sign test statistics in order to monitor the process variability. The scheme established by Amin et al. (1995) is user friendly. Due to their nonparametric nature no assumptions for the underlying distribution are required, while their schemes are proved to be more capable than the respective parametric ones when the process distributional model is heavy tailed.

Let us denote by $X_{i 1}, X_{i 2}, \ldots, X_{i n}, i=1,2, \ldots$ a group of magnitude $n$ consisting of observations which are drawn randomly at regular intervals from the process. The distributional model of the underlying production is presumed to be continuous with median $M$ and the interest focuses on detecting possible shifts of its median from a pre-specified target value $M_{0}$. The main idea of the monitoring scheme established by Amin et al. (1995) is based on the exploitation of the information included inside the samples up to the current time. In their article, all comparisons among the proposed monitoring scheme and other competitive charts rely on the corresponding Average Run Length values.

Generally speaking, control schemes which rely on the sign statistic call for comparisons of each observation against the target (or control) value $M_{0}$ and the recording of the amount of observations which are located below and above $M_{0}$ respectively. Denoting by $\operatorname{sign}(a)$ the sign of number $a$, Amin et al. (1995) utilized the following test statistic

$S N_{i}=\sum_{j=1}^{n} \operatorname{sign}\left(X_{i j}-M_{0}\right), i=1,2, \ldots$

It is straightforward that the sign statistic defined in Eq. (7) is computed for each random sample separately and corresponds to the diversion between the amount of observations located above $M_{0}$ and the respective number of observations positioned below $M_{0}$. The CUSUM schemes established by Amin et al. (1995) can be constructed for detecting either positive or negative deviations from the IC median value. More precisely, the one-sided scheme gives an alarm at the first $d$ samples if one of the following ensues 
$\sum_{i=1}^{d}\left(S N_{i}-s\right)-\min _{0 \leq m \leq d} \sum_{i=1}^{m}\left(S R_{i}-s\right) \geq h$

Or

$\max _{0 \leq m \leq d} \sum_{i=1}^{m}\left(S N_{i}+s\right)-\sum_{i=1}^{d}\left(S R_{i}+s\right) \geq h$,

where $s, h$ are positive design parameters of the corresponding scheme. Note that the firstmentioned condition appeared in Eq. (8) is activated whenever a possible positive shift from the IC target value is suspected, while the second one is more suitable for dealing with plausible negative shifts. As mentioned by Amin et al. (1995), the corresponding two-sided scheme is readily deduced if we take into consideration both conditions stated in Eq. (8), namely their two-sided control chart is expected to signal at the first $d$ samples if either of the one-sided charts give an alarm. A different approach for implementing the CUSUM scheme established by Amin et al. (1995) is offered by the so-called graphical V-mask scheme (see, e.g. Van Dobben de Bruyn, 1968).

Amin et al. (1995) pointed out that the random quantity $T_{i}=\left(S N_{i}+n\right) / 2$, which corresponds to the amount of positive signs throughout the observations of the $i$-th random sample, could be an alternative test statistic for their CUSUM procedure. It is evident that the random variable $T_{i}$ follows a Binomial distribution with parameters $n$ and $p=P\left(X_{i j}>M_{0}\right)$. Consequently, one may restate the proposed chart by Amin et al. (1995) by utlizing the test statistic $T_{i}$ instead of $S N_{i}$.

The ARL of the corresponding CUSUM scheme could be determined in terms of design parameters $s$ and $h$. For instance, one may optimize the OOC behavior of the control chart, given that its IC performance is pre-specified. In other words, one may construct the CUSUM control chart such that it achieves a desirable IC $A R L$ and it performs simultaneously the minimum OOC $A R L$. Some interesting thoughts on the aforementioned optimization issue are offered by Reynolds (1975).

Amin et al. (1995) recommended the usage of distribution-free schemes based on signs, for heavytailed or asymmetric distributions. They also argued that their proposal seems to offer an alluring framework when relatively large samples, e.g. samples of size greater than 6 , for detecting possible small shifts.

\subsection{A Distribution-Free CUSUM Mean Control Chart}

Yang and Cheng (2011) established a distribution-free CUSUM mean scheme for monitoring possible small mean changes in the underlying distribution. In their framework, no assumptions referring to the process distribution is required, while an extensive numerical experimentation reveals that the proposed scheme is quite favorable versus other existing competitive charts, especially for unascertained or non-normal distributions.

Let us denote by $X_{i 1}, X_{i 2}, \ldots, X_{i n}, i=1,2, \ldots$ a group of magnitude $n$ consisting of observations which are drawn randomly and independently from the production. We next denote by $\mu$ the process mean and by $p$ the probability that an observed value taken randomly from the process is greater than $\mu$. Presuming that the process is IC, the probability $p$ is said to be equal to $p_{0}$. Conversely, when a change in the process mean has occurred, the corresponding probability equals to $p_{1}$. 
Based on the observed values drawn from the process, Yang and Cheng (2011) formed the differences $Y_{i j}=X_{i j}-\mu, i=1,2, \ldots$ and defined the following sign statistic

$M_{i}=\sum_{j=1}^{n} I\left(Y_{i j}>0\right), j=1,2, \ldots, n$

where

$I\left(Y_{i j}>0\right)=\left\{\begin{array}{l}1, \text { if } Y_{i j}>0 \\ 0, \text { otherwise }\end{array}, j=1,2, \ldots, n\right.$,

while $i$ is a positive integer.

It is straightforward that the quantity $M_{i}$, which corresponds to the number of non-negative $Y_{i j}$ inside the $i$-th random sample, follows a Binomial distribution with parameters $n$ and $p_{0}$ for an IC process. Yang and Cheng (2011) pointed out that instead of supervising slight process mean changes, it is similar to look for slight shifts in probability $p$. Their proposed two-sided CUSUM nonparametric control chart utilizes two different monitoring statistics. If $\Delta=\left|p_{0}-p_{1}\right|, \Delta>0$, then Yang and Cheng (2011) defined the following CUSUM test statistics

$C_{i}^{+}=\max \left(0, C_{i-1}^{+}+M_{i}-\left(n p_{0}+k_{1}\right)\right), i=1,2, \ldots$

and

$C_{i}^{-}=\min \left(0, C_{i-1}^{-}-\left(n p_{0}-k_{1}\right)+M_{i}\right), i=1,2, \ldots$

where $M_{i}$ corresponds to the number of non-negative $Y_{i j}, k_{1}=n \Delta / 2$ and the starting values equal to zero, e.g. $C_{0}^{+}=0, C_{0}^{-}=0$. Note that both monitoring statistics defined in Eq. (11) and (12) are plotted on the same chart, whose upper and lower control limit is denoted by $H_{1}$ and $-H_{1}$ respectively. The CUSUM scheme introduced by Yang and Cheng (2011) gives an OOC alarm whenever at least one the following occurs

$C_{i}^{+} \geq H_{1}$ or $C_{i}^{-} \leq-H_{1}$.

It is evident that the design constants $k_{1}$ and $H_{1}$ of the respective chart are appropriately determined in order a pre-determined IC or OOC performance level is accomplished. As Yang and Cheng (2011) mentioned, in order to calculate the Average Run Length of their two-sided control scheme, the upper and lower half of the chart should be considered separately. In fact, the upper half $\left(\mathrm{CUSUM}^{+}\right.$, hereafter) is responsible for tracking down plausible upward shifts of the process, while the lower half (CUSUM-, hereafter) is activated for monitoring possible downward changes. Denoting by $A R L^{+}\left(A R L^{-}\right)$the Average Run Length of the CUSUM ${ }^{+}\left(\mathrm{CUSUM}^{-}\right)$, the overall $A R L$ of the corresponding CUSUM scheme is expressed as Hawkins (1992). 


$$
A R L=\frac{1}{1 / A R L^{+}+1 / A R L^{-}} .
$$

Yang and Cheng (2011) managed to determine the $A R L^{+}$and $A R L^{-}$values appeared in the above equation, by the aid of an appropriate Markov chain approach (see, e.g. Lucas and Saccucci, 1990). Yang and Cheng (2011) provided some numerical results for emphasizing the efficiency of the proposed CUSUM scheme in tracking down plausible changes of the process, even if the process distribution is skewed. Generally speaking, the CUSUM chart established by Yang and Cheng seems to constitute an attractive alternative to traditional Shewhart-type or CUSUM-type monitoring schemes when the underlying distribution is unascertained or non-normal.

\subsection{A Nonparametric CUSUM Sign Control Chart Using RSS}

Abid et al. (2017) established a CUSUM sign scheme for tracking down plausible deviations from the process mean applying the so-called RSS. According to their simulation study, the proposed CUSUM sign monitoring scheme seems to outperform the modified distribution-free CUSUM sign scheme and the parametric CUSUM scheme utilizing simple random sampling framework.

Abid et al. (2017) proposed the construction of CUSUM-type monitoring scheme by the aid of the RSS method. According to the aforementioned sampling technique, a group of size $n$ is collected randomly from the production and afterwards an expert is due to rank its units. The observation having the minimum rank is located and selected for the quantification. The remaining $(n-1)$ units are backtracked and a second random sample of size $n$ is collected. After ranking the observations of the second sample, the observation with the second smallest rank is detected, measured, and the unused observations are backtracked once again. The aforementioned procedure is replicated until $n$ ordered observations have been measured and that completes the first cycle. After accomplishing $m$ separate cycles, one may obtain a ranked set sample consisting of $\mathrm{rm}$ observations. For some recent advances on the RSS procedure, which has been introduced by McIntyre (1952), the interested reader is referred to Frey and Zhang (2021), Koshti and Kamalja (2021).

Let us denote by $X_{(1) 1}, X_{(2) 1}, \ldots, X_{(n) 1}, X_{(1) 2}, X_{(2) 2}, \ldots, X_{(n) 2}, \ldots, X_{(1) m}, X_{(2) m}, \ldots, X_{(n) m}$ a ranked set random sample of size $n$ with $m$ cycles, consisting of observations which are drawn independently from the process. We next denote by $\mu$ the target value and by $p$ the probability that an observed value taken randomly from the process is greater than $\mu$. Presuming that the process is IC, the probability $p$ is said to be equal to $p_{0}$. Conversely, when a change in the process mean has occurred, the corresponding probability equals to $p_{1}$.

Based on the observed values drawn from the process, Abid et al. (2017) considered the following sign statistic (Hettmansperger, 1995).

$$
M_{R S S}^{+}=\sum_{j=i}^{r} \sum_{i=1}^{m} I\left(X_{(j) i}-\mu>0\right)
$$

where

$$
I\left(X_{(j) i}-\mu>0\right)=\left\{\begin{array}{l}
1, \text { if } X_{(j) i}-\mu>0 \\
0, \text { otherwise }
\end{array}\right.
$$


Their proposed two-sided CUSUM nonparametric control chart utilizes two different monitoring statistics. If $\Delta=\left|p_{0}-p_{1}\right|, \Delta>0$, then Abid et al. (2017) defined the following CUSUM test statistics

$$
C_{t}^{+}=\max \left(0, C_{t-1}^{+}+M_{R S S(t)}^{+}-\left(n p_{0}+k\right)\right), t=1,2, \ldots
$$

and

$$
C_{t}^{-}=\min \left(0, C_{t-1}^{-}-\left(n p_{0}-k\right)+M_{R S S(t)}^{+}\right), t=1,2, \ldots
$$

where $M_{R S S(t)}^{+}$corresponds to the number of non-negative $X_{(j) i}-\mu$ at time $t, n p_{0}$ coincides to the mean value, $k$ is a reference value with $k=n \Delta / 2$ and the starting values are normally to be equal to zero, e.g. $C_{0}^{+}=0, C_{0}^{-}=0$. Note that both monitoring statistics defined in Eq. (17) and (18) are plotted on the same chart, whose upper and lower control limit is denoted by $H$ and $-H$ respectively. The CUSUM scheme introduced by Abid et al. (2017) produces an alarm whenever at least one the following occurs

$C_{t}^{+} \geq H$ or $C_{t}^{-} \leq-H$

It is evident that the design constants $k$ and $H$ of the corresponding chart are appropriately defined such that a pre-determined IC or OOC behavior is accomplished. It is noticeable that several wellknown nonparametric testing procedures have been investigated in terms of the RSS (Bohn and Wolfe, 1992; Bohn and Wolfe, 1994; Hettmansperger, 1995). At the same time, some interesting applications of the RSS method on the development of parametric control charts are provided by Abujiya and Muttlak (2004), Mehmood et al. (2013), Haq et al. (2015). The numerical comparisons accomplished by Abid et al. (2017) confirm that their scheme is capable for tracking down slight and big changes in the process mean immediately versus competitive schemes.

\subsection{A Nonparametric CUSUM Control Chart Based on Ranks and RSS}

Abid et al. (2018) established a nonparametric scheme, which relies on the Wilcoxon signed rank statistic and the so-called RSS. Since RSS has been discussed in Sub-Section 2.4 of the present manuscript in some detail, we avoid illustrating its framework once again throughout the lines of the present section.

A variety of distributions, namely the Laplace distribution, the Logistic, Normal or Student's tdistribution, is utilized to study the behavior of the CUSUM monitoring scheme. According to the numerical results provided by Abid et al. (2018), the proposed chart seems to outperform other competitive control charts.

Let us suppose that a random sample of size $n$ is drawn from the process in order to monitor the process location $\theta_{0}$. Since Abid et al. (2018), implemented the RSS with $m$ cycles, the proposed testing procedure is based on the quantity $R_{(p h) i}^{+}$, which corresponds to the within-group absolute 
rank of deviations from $\theta_{0}$, e.g. $R_{(p h) i}^{+}=\left|X_{(p h) i}-\theta_{0}\right|, h=1,2, \ldots, n$. The nonparametric signed rank statistic under the RSS framework is given by Kim and Kim (1996).

$S R_{R S S_{p}}=\sum_{h=1}^{n} \sum_{i=1}^{m} \operatorname{sign}\left(X_{(p h) i}-\theta_{0}\right) R_{(p h) i}^{+}, p=1,2, \ldots, h=1,2, \ldots, n$ and $i=1,2, \ldots, m$

while

$$
\operatorname{sign}\left(X_{(p h) i}-\theta_{0}\right)= \begin{cases}1, & \text { if } X_{(p h) i}-\theta_{0}>0 \\ 0, & \text { if } X_{(p h) i}-\theta_{0}=0 \\ -1, & \text { if } X_{(p h) i}-\theta_{0}<0 .\end{cases}
$$

The expected value and the variance of the test statistic defined in Eq. (20) are given by

$$
E\left(S R_{R S S}\right)=0, \operatorname{Var}\left(S R_{R S S}\right)=(n m(m+1)(2 m+1) / 6) \delta_{0}^{2},
$$

where

$$
\delta_{0}^{2}=1-(4 / n) \sum_{h=1}^{n}\left(F_{h}(0)-1 / 2\right)^{2},
$$

while $F_{h}(0)$ can be computed by the aid of the incomplete beta integral, e.g.

$$
F_{h}(0)=\frac{r !}{(h-1) !(r-h) !} \int_{-\infty}^{0} F^{h-1}(u)(1-F(u))^{r-h} f(u) d u
$$

Abid et al. (2018) proposed a CUSUM nonparametric control chart, which utilizes the following different monitoring statistics

$$
C_{p}^{+}=\max \left(0, C_{p-1}^{+}+S R_{R S S_{p}}-k\right), p=1,2, \ldots
$$

and

$C_{p}^{-}=\max \left(0, C_{p-1}^{-}-k-S R_{R S S_{p}}\right), p=1,2, \ldots$

where $S R_{R S S_{p}}$ is defined in Eq. (20) and $k$ is the reference value of the CUSUM chart. The abovementioned statistics are plotted against a single control limit, say $h$, and the proposed control chart produces an OOC signal whenever at least one of the statistics defined in Eq. (24) and (25) are plotted outside of the control limit. It is straightforward that the design parameters $k$ and $h$ of the corresponding chart are appropriately determined for accomplishing the desired level of performance. 
Abid et al. (2018) assessed their proposed CUSUM chart for both IC and OOC processes based on its run length distribution. More precisely, they carried out a Monte-Carlo simulation study for calculating the run length values of the proposed nonparametric scheme and provided several numerical comparisons versus other existing distribution-free control charts. Their general conclusion was that the particular chart is capable enough in detecting slight and big location changes.

\subsection{A Nonparametric CUSUM Control Chart Based on Deciles}

Shirke and Barale (2018) established a nonparametric cumulative sum control chart for process dispersion based on the sign statistic using in-control deciles. The particular scheme can be viewed as a modification of the control chart established by Amin et al. (1995), which has been discussed in previous subsection of the present manuscript.

Let $X$ denote the quality characteristic of interest with variance $\sigma^{2}$. Under the presumption that the process is IC, the variance of $X$ equals to $\sigma_{0}^{2}$, while if a shift is present then the corresponding variance is denoted by $\sigma_{1}^{2}$. For monitoring the underlying process, random samples of size $n$ are drawn, say $\left(X_{i 1}, X_{i 2}, \ldots, X_{i n}\right), i=1,2, \ldots$

Given the observed values drawn from the process, Shirke and Barale (2018) defined the following deciles-based statistic

$U_{i j}=\left\{\begin{array}{l}1, X_{i j} \leq D_{2} \text { or } X_{i j} \geq D_{8} \\ 0, X_{i j}>D_{2} \text { or } X_{i j}<D_{8}\end{array}\right.$

where $D_{2}$ and $D_{8}$ correspond to the 2nd and 8th decile when the process is IC respectively. Considering the quantity $U_{i}=\sum_{j=1}^{n} U_{i j}$, it is straightforward that $U_{i}$ follows the Binomial distribution with parameters $n$ and $p$, where $p=P\left(X_{i j} \leq D_{2}\right.$ or $\left.X_{i j} \geq D_{8} \mid \sigma=\delta \sigma_{0}\right)$. Note that the probability $p$ is denoted by $p_{0}\left(p_{1}\right)$ when the process is assumed to be IC (OOC). As Shirke and Barale (2018) argued, small shifts in process dispersion can be monitored by the aid of the corresponding proportion of those observations which fall in the tails. More specifically, Shirke and Barale (2018) defined the following CUSUM test statistics

$C_{t}^{+}=\max \left(0, C_{t-1}^{+}+U_{i}-\left(n p_{0}+k\right)\right), i=1,2, \ldots$

and

$C_{t}^{-}=\max \left(0, C_{t-1}^{-}+\left(n p_{0}-k\right)-U_{i}\right), i=1,2, \ldots$

where $k$ is a reference value with $k=n\left|p_{0}-p_{1}\right| / 2$ and the starting values are normally to be equal to zero, e.g. $C_{0}^{+}=0, C_{0}^{-}=0$. The proposed monitoring scheme produces an OOC signal if at least 
one of the following is satisfied $C_{i}^{+}>h, C_{i}^{-}>h$. In fact, the first condition is activated when an increase in process dispersion is suspected, while the second one is suitable for monitoring possible negative shifts in the process dispersion. Generally speaking, the CUSUM control chart introduced by Shirke and Barale (2018), seems to be capable for detecting small shifts in process dispersion and provides an excellent alternative to traditional CUSUM-type schemes, especially when the process distribution is unknown.

\section{Distribution-Free CUSUM Control Charts Based on Order Statistics}

In the present section, we refer to univariate nonparametric schemes, which rely on the order statistics and follow the general CUSUM- framework. It is evident that order statistics (such as the median) have been already implemented for establishing Shewhart-type distribution-free schemes (Janacek and Meikle, 1997; Triantafyllou, 2018; Triantafyllou, 2019). However, our study focuses on presenting the respective CUSUM-type advances. We next illustrate a detailed review of nonparametric univariate monitoring schemes based on order statistics, namely of these members of the $C N C C$ class which utilize as monitoring statistic one (or more) order statistic. The order of appearance of the included publications is determined to be chronological for the purpose of setting up a comprehensible and well-structured flow.

\subsection{A One-Sided Distribution-Free CUSUM Scheme Based on Exceedance Statistics} Mukherjee et al. (2013) proposed a nonparametric CUSUM scheme which utilizes the exceedance statistics. Its efficiency in tracking over a location change in the unascertained distribution seems to be greater than the corresponding of the rank-based charts, in particular for heavy-tailed or positively asymmetric distributions.

Generally speaking, the precedence and the exceedance testing procedure was introduced by Nelson (1963) for detecting, as early as possible, differences between two samples placed at the same time on a life test. In simple words, the precedence (exceedance) statistic relies on the amount of observed values from the one of the samples that precede (exceed) a pre-determined (say the $r$ th) order statistic of the other sample. Since the precedence statistic is linearly connected to the exceedance one, it is readily obtained that the aforementioned test statistics are equivalent. For more details about precedence-type tests and their applications in life-testing issues designed for reaching conclusions at early stages of an experiment, the excellent work provided by Balakrishnan and $\mathrm{Ng}$ (2006) is highly recommended.

Let us denote by $X_{1}, X_{2}, \ldots, X_{m}$ a reference sample of size $m$, which is drawn from the IC distribution $F$. In addition, test samples of magnitude $n$ from distribution $G$, say $X_{j 1}, X_{j 2}, \ldots, X_{j n}, j=1,2, \ldots$, are collected so that the characterization of the process as IC or OOC, e.g. $F=G$ or $F \neq G$, can be accomplished. Denoting by $U_{j, r}$ the amount of exceedances, namely the amount of units from the test sample that exceeds the $r$-th order statistic of the reference sample, it is easily deduced that, given $X_{(r)}=x_{(r)}$, the variable $U_{j, r}$ is Binomial with parameters $n$ and $p_{r}$, where $p_{r}=P\left(Y>X_{(r)} \mid X_{(r)}\right)$ is the so-called exceedance probability. Consequently, Mukherjee et al. (2013) utilized a CUSUM control chart which rely on the quantities $U_{j, r}$ 's in order to supervise the location by the aid of the corresponding exceedance probabilities. More precisely, they defined the following CUSUM-type statistic. 
$C_{j}=\max \left(0, C_{j-1}+\left(U_{j, r}-n d\right)-k\right), j=1,2,3, \ldots$

where $d=(m-r+1) /(m+1)$ corresponds to the unconditional IC probability $p_{r}$ and $k$ is the nonnegative reference value. Note that the starting value $C_{0}$ is assumed to be zero. It goes without saying that their upper one-sided CUSUM nonparametric control scheme produces an OOC signal whenever $C_{j}$ is greater than a positive-valued parameter $H$, which is appropriately determined so that a pre-determined efficiency is accomplished.

Mukherjee et al. (2013) studied the run length of the resulting CUSUM nonparametric scheme monitoring scheme by the aid of a Markov chain technique (Brook and Evans, 1972; Gan, 1993). The unconditional $A R L$ of their scheme was determined by the aid of the order statistic $X_{(r)}$.

It is evident that in case of collecting the data in a common way, the exceedance schemes are beneficial due to their ability to be implemented prematurely, resulting in rescuing time and supplies. Thus, practically speaking, minimal presumptions, resilience of the IC run-length distribution, and OOC behavior, the resulting scheme seems to be quite competitive in practical situations. Generally speaking, their Phase II CUSUM control chart seems to provide nice performance for tracking down an increasing change in the location parameter of the underlying process distributional model.

\subsection{A Two-Sided Nonparametric CUSUM Control Chart Based on Exceedance Statistics}

Graham et al. (2014) studied CUSUM schemes for supervising the unknown location parameter of the underlying process. Their study focuses on testing procedures based on the exceedance statistic defined in the previous subsection of the present manuscript. Contrary to previous works developed in this direction, the present article introduces a two-sided CUSUM scheme, while the median run length (Khoo et al., 2011) is utilized as an efficiency measure instead of the usual average value.

Recalling the variable $U_{j, r}$ defined earlier, Graham et al. (2014) proposed the following monitoring CUSUM-type statistics

$C_{j}^{+}=\max \left(0, C_{j-1}^{+}+\left(U_{j, r}-n d\right)-k\right), j=1,2,3, \ldots$

and

$C_{j}^{-}=\min \left(0, C_{j-1}^{-}+\left(U_{j, r}-n d\right)+k\right), j=1,2,3, \ldots$

with starting values equal to zero and a non-negative reference value $k$. Their monitoring scheme produces an OOC signal if $C_{j}^{+}>H$ or $C_{j}^{-}<-H$, where $H>0$. Graham et al. (2014) investigated systematically the performance of their proposed chart based on several percentiles, while the effect of the choice of the reference value is also discussed. According to their numerical results, it seems that the exceedance CUSUM chart based on higher percentiles outperforms in a lot of cases considered. Additionally, the usage of $75^{\text {th }}$ percentile of the reference sample as the corresponding 
reference parameter, seems to offer an intriguing behavior of the resulting chart for tracking down large location changes.

\subsection{A Nonparametric CUSUM Control Chart Based on the Extended Median Test}

Brown and Schaffer (2020) proposed a CUSUM charting technique for multiple stream processes, which relies on an alteration of the traditional extended median test. When a lot of identical processes with identical target values are supervised, a variety of approaches has been proposed in the literature. For instance, Runger et al. (1996) argued that a multivariate monitoring scheme should be constructed, while Meneces et al. (2008) introduced implementing a Shewhart-type scheme for each stream.

Brown and Schaffer (2020) utilized as monitoring statistic, an alteration of the extended median test, which was first studied by Conover (1998). In fact, the classical extended median test can be viewed as a parallel nonparametric approach to the traditional one-way Analysis of Variance parametric model. Throughout the lines of the manuscript of Brown and Schaffer (2020), it is presumed that streams are considered to be independently distributed binomial random variables, while the sample size drawn from each flow is supposed to be adequate for implementing the corresponding asymptotic distribution.

Let $X_{i 1 t}, X_{i 2 t}, \ldots, X_{i n_{i} t}$ be a random group of magnitude $n_{i}$ drawn from the $i$-th flow at time $t$, where $t=1,2, \ldots$ and $i=1,2, \ldots, Q$. Assuming that all supervised flows share an identical median $M_{0}$, we denote by $L_{1 t}, L_{2 t}, \ldots, L_{Q t}$ the corresponding frequencies of those units being equal to or greater than $M_{0}$. Given that the process is IC, the frequencies $O_{i t}$ are Binomial random variables with parameters $n_{i t}$ and $p_{0}=0.5$. Consequently, it is straightforward that the corresponding standardized random variable is given by

$Z_{i t}=\frac{L_{i t}-n_{i t} p_{0}}{\sqrt{n_{i t} p_{0}\left(1-p_{0}\right)}}, t>0$ and $i$ corresponds to a positive integer.

Since the dispersion of a sum of independently distributed random quantities coincides to the total sum of each corresponding random variable's dispersion component, the following ensues

$E M T_{t}=\sum_{i=1}^{Q} Z_{i t} \sim$ Normal distribution $N(0, Q)$

Based on the test statistic defined above, Brown and Schaffer (2020) introduced the following CUSUM statistic

$$
S_{t}=\sum_{j=1}^{t} E M T_{j}=S_{t-1}+E M T_{t}
$$


where $S_{0}=0$. Conditionally on $S_{t-1}$, they also argued that $S_{t}$ is normally distributed with parameters $s_{t-1}$ and $C$. Consequently, the following hold true

$U C L_{t}=s_{t-1}+\delta \sqrt{Q}, \quad L C L_{t}=s_{t-1}-\delta \sqrt{Q}$,

where $\delta$ is suitably determined so that a desired efficiency is accomplished. The simulation study carried out by Brown and Schaffer (2020) reveals that, under several non-normal distributional models, their monitoring scheme seems to have superior performance in comparison with other competitive charts. For the particular CUSUM nonparametric control chart, the alternative distribution of the monitoring statistic is provided by Brown (2020) and consequently the corresponding statistical power can be estimated.

\section{Distribution-Free CUSUM Control Charts Based on Rank Statistics}

Throughout the lines of the present part, univariate nonparametric schemes, which rely on ranks and follow the general CUSUM framework are discussed. It is evident that rank-based statistics have been already utilized for constructing Shewhart-type distribution-free schemes (Triantafyllou and Panayiotou, 2020; Triantafyllou, 2021). However, our study aims at discussing the corresponding CUSUM-type advances. We next provide a detailed review of nonparametric univariate monitoring schemes based on ranks, namely of these members of the $C N C C$ class which employ several rank-based statistics. The set-up of presentation of the included articles is determined to be chronological for the purpose of setting up a comprehensible and well-structured flow.

\subsection{A Nonparametric CUSUM Control Chart Based on Wilcoxon Rank-Sum Statistics}

Li et al. (2010) introduced an analog of CUSUM control chart based on the Wilcoxon rank-sum statistic for detecting possible shifts of the mean of the underlying process. Their study relies on the run length distribution of the resulting nonparametric control chart. According to their numerical results, the proposed distribution-free CUSUM scheme outperforms its competitors when the underlying distribution is non-normal, while it performs almost the same for normally distributed data.

It is evident that the Wilcoxon rank-sum statistic offers an equivalent testing procedure with the corresponding one provided by the so-called Mann Whitney statistic (Manoukian, 1986). The Wilcoxon rank-sum statistic and its modifications have been already utilized for constructing Shewhart-type monitoring schemes (Balakrishnan et al., 2009; Triantafyllou and Panayiotou, 2020; Triantafyllou, 2021).

Let $X_{1}, X_{2}, \ldots, X_{m}$ be a group of magnitude $m$ drawn randomly from the IC production. The $X$ observations form the so-called reference sample of the charting procedure. Afterwards, several test samples of size $n$, e.g. $Y_{1}, Y_{2}, \ldots, Y_{n}$ are drawn from the process and a conclusion referring to status of the process is about to be reached. For determining the Wilcoxon rank-sum statistic (see, e.g. Wilcoxon, 1945), one may arrange all $(m+n)$ observations in ascending order and put them in a pooled sample. Afterwards, define $a_{i}$ such that 
$a_{i}=\left\{\begin{array}{l}1, \text { if a } X-\text { observation is placed in the } i-\text { th entry of the pooled sample } \\ 0, \text { if a } Y-\text { observation is placed in the } i-\text { th entry of the pooled sample }\end{array}\right.$

The Wilcoxon rank-sum statistic is now given by

$W=\sum_{i=1}^{m+n} i a_{i}$

while its mean and variance under the identical distributions' assumption, can be expressed as

$$
E(W)=\frac{n(m+n+1)}{2}, V(W)=\frac{m n(m+n+1)}{12}
$$

respectively. Based on the above-mentioned argumentation, Li et al. (2010) proposed the following CUSUM-type statistics

$$
C_{i}^{+}=\max \left(0, C_{i-1}^{+}+\left(W_{i}-n(m+n+1) / 2\right)-k\right), i=1,2,3, \ldots
$$

and

$$
\left.C_{i}^{-}=\max \left(0, C_{i-1}^{-}+n(m+n+1) / 2\right)-k-W_{i}\right), i=1,2,3, \ldots
$$

where $k$ is the reference parameter of the CUSUM scheme. It goes without saying that the CUSUM scheme produces an OOC alarm if $C_{i}^{+}$or $C_{i}^{-}$is equal to or exceeds the limit $h$.

Since all test samples are compared to the same reference sample, it is evident that the observed values of the plotted statistic shall be correlated. This correlation is a consequence of the uncertainty of the reference sample with respect to the real distribution. For overcoming the correlation issue, $\mathrm{Li}$ et al. (2010) followed a conditioning argumentation. Moreover, the conditional $R L$ - distribution for their upper-sided CUSUM nonparametric control scheme is computed via the following recursive approach (Page (1954)).

$P\left(R L=1 \mid u, X_{1}, X_{2}, \ldots, X_{m}\right)=P\left(W \geq n(m+n+1) / 2+k+h-u \mid u, X_{1}, X_{2}, \ldots, X_{m}\right)$

and

$$
\begin{aligned}
& P\left(R L=t \mid u, X_{1}, X_{2}, \ldots, X_{m}\right)= P\left(R L=t-1 \mid u=0, X_{1}, X_{2}, \ldots, X_{m}\right) \\
& \times P\left(W \leq n(m+n+1) / 2+k-u \mid u, X_{1}, X_{2}, \ldots, X_{m}\right) \\
&+\sum_{v \in V}\left(P\left(R L=t-1 \mid v, X_{1}, X_{2}, \ldots, X_{m}\right)\right) \cdot P\left(W=v+n(m+n+1) / 2+k-u \mid u, X_{1}, X_{2}, \ldots, X_{m}\right)
\end{aligned}
$$

where $V$ contains all possible values for $C_{i}^{+}$. Similarly, Li et al. (2010) obtained a recursive scheme for the conditional $R L$-distribution of their lower-sided CUSUM control chart

$$
P\left(R L=1 \mid u, X_{1}, X_{2}, \ldots, X_{m}\right)=P\left(W \geq k-u-n(m+n+1) / 2+h \mid u, X_{1}, X_{2}, \ldots, X_{m}\right)
$$


and

$$
\begin{aligned}
& P\left(R L=t \mid u, X_{1}, X_{2}, \ldots, X_{m}\right)= P\left(R L=t-1 \mid u=0, X_{1}, X_{2}, \ldots, X_{m}\right) \\
& \times P\left(W \geq u+n(m+n+1) / 2-k \mid u, X_{1}, X_{2}, \ldots, X_{m}\right) \\
&+\sum_{v \in V}\left(P\left(R L=t-1 \mid v, X_{1}, X_{2}, \ldots, X_{m}\right)\right) \cdot P\left(W=u+n(m+n+1) / 2-k-v \mid u, X_{1}, X_{2}, \ldots, X_{m}\right)
\end{aligned}
$$

Some interesting remarks for the role that the sampling magnitude plays upon the efficiency of the resulting chart are concluded and a few practical guidelines for optimizing its design are also provided. As Li et al. (2010) argued, the larger the reference sample size, the less unsureness and the better the performance. Additionally, if the reference sample size is pre-specified, smaller (larger) test sample size is preferable for detecting small (large) shifts.

\subsection{A Sequential Rank-Based Nonparametric CUSUM Control Chart}

Liu et al. (2014) established a sequential rank-based adaptive nonparametric CUSUM monitoring scheme for detecting possible shifts of the location parameter of the underlying distribution. Due to its self-starting nature, the proposed chart is capable to monitor a process at its early stage and that makes the new scheme quite advantageous. A pioneer work on the topic was provided by McDonald (1990), where a CUSUM procedure based on the so-called sequential ranks for individual observations was considered.

Suppose that the observations $X_{t}$ are drawn independently from the next change-point schedule (Hawkins and Deng, 2010).

$x_{t}=\left\{\begin{array}{l}F\left(x, \mu_{0}\right), \text { for } t=1,2, \ldots, \tau \\ F\left(x, \mu_{1}\right), \text { for } t=\tau+1, \tau+2, \ldots,\end{array}\right.$

while $\tau$ denotes the unascertained change point parameter, $\mu_{0}\left(\mu_{1}\right)$ corresponds to the IC (OOC) location parameter of the process, while $F$ denotes its continuous distribution function.

We next denote by $R_{t}$ the $t$-th sequential rank, namely the rank of observed value $x_{t}$ among the observations $x_{1}, x_{2}, \ldots, x_{t}$. which can be expressed as

$R_{t}=\sum_{j=1}^{t} I\left(x_{t} \geq x_{j}\right)$

The mean and variance of $R_{t}$, under the assumption that the process is IC, are given by

$E\left(R_{t}\right)=(t+1) / 2, V\left(R_{t}\right)=(t+1)(t-1) / 12$

respectively. Since the distribution of $R_{t}$ varies as $t$ increases, it seems that the corresponding standardized values should be considered. By defining the $t$-th standardized sequential rank as 
$R_{t}^{*}=\frac{R_{t}-E\left(R_{t}\right)}{\sqrt{V\left(R_{t}\right)}}, t \geq 2$,

Liu et al. (2014) introduced the one-sided upper and lower adaptive nonparametric CUSUM control charts, whose monitoring statistics are given by

$$
S_{t}^{+}=\max \left(0, S_{t-1}^{+}+\left(R_{t}^{*}-\hat{\delta}_{t}^{+} / 2\right) / h\left(\hat{\delta}_{t}^{+} / 2\right)\right)
$$

and

$$
S_{t}^{-}=\min \left(0, S_{t-1}^{-}+\left(R_{t}^{*}-\hat{\delta}_{t}^{-} / 2\right) / h\left(\hat{\delta}_{t}^{-} / 2\right)\right)
$$

respectively. Note that $\hat{\delta}_{t}^{+}=\max \left(Y_{t}, \delta_{0}\right)$ and $\hat{\delta}_{t}^{-}=\min \left(Y_{t},-\delta_{0}\right)$, where $Y_{t}$ estimates the change in $R_{t}^{*}$ and $\delta_{0}$ corresponds to a change of a minimum size of concern for a beforehand disclosure. Throughout the lines of their manuscript, Liu et al. (2014) plotted both statistics defined in Eq. (47) and (48) in a time sequence versus the control limits $h_{1}, h_{2}$ and an OOC signal is produced whenever $S_{t}^{+}>h_{1}$ or $S_{t}^{-}<h_{2}$. Assuming that $h_{1}=h_{2}$, then if the monitoring statistic $S_{t}=\max \left(S_{t}^{+},\left|S_{t}^{-}\right|\right)$ is implemented, the corresponding two-sided CUSUM scheme is emerged.

Based on the numerical experimentation carried out by Liu et al. (2014), some practical remarks have been concluded. For instance, it has been numerically verified that as more IC units are drawn, the proposed scheme becomes more capable for tracking over shifts. Moreover, the proposed CUSUM chart is capable to track over a wider variety of mean changes, by arranging its reference constants with respect to the knowledge based on the ongoing phase, compared to the classical distribution-free schemes. It also exhibits a good performance for big changes without depending on the distributional model.

\subsection{A Sequential Rank-Based Distribution-Free CUSUM Scheme}

Liu et al. (2015) introduced a dual distribution-free CUSUM control chart which rely on ranks for detecting the persistent shifts in location parameter. A simulation procedure accomplished by the authors exposes that the corresponding scheme not only exhibits a robust performance for several distributional models, but also is capable for tracking over various magnitudes of changes.

Suppose that the observations $X_{t}$ are drawn independently over time from the change-point model appeared in Eq. (43) of the present manuscript. Liu et al. (2015) exploited the $t$-th sequential rank of the abovementioned observations, in order to build up a one-sided nonparametric CUSUM control chart. Since the $t$-th standardized sequential rank defined in Eq. (46) takes on values from the following set

$$
\left\{\frac{i-(t+1) / 2}{\sqrt{(t+1)(t-1) / 12}}, i=1,2, \ldots, t\right\},
$$

a straightforward chart based on $R_{t}^{*}$ utilizes the following CUSUM-type statistic 
$S_{t}=\max \left(S_{t}^{+},\left|S_{t}^{-}\right|\right)$, where $S_{t}^{+}=\max \left(0, S_{t-1}^{+}+R_{t}^{*}-k\right)$ and

$S_{t}^{-}=\min \left(0, S_{t-1}^{-}+R_{t}^{*}+k\right)$.

For a constant $k$, the proposed scheme produces an alarm whenever $S_{t}>h$, where $h>0$. However, the one-sided common CUSUM statistic can be easily optimized for detecting a location shift only under standard normal distribution (Bagshaw and Johnson, 1975). In fact, under unascertained distributional model, there is no certainty that the corresponding optimization problem has a solution. Consequently, a single CUSUM statistic is not expected to exhibit an optimal behavior over a span of anticipated location changes. To face up to the above-mentioned drawback, Liu et al. (2015) implemented two distribution-free CUSUM schemes simultaneously with nonidentical degree of retention by utilizing CUSUM-type statistics with nonidentical $k$ values. It is straightforward that an alarm is generated whenever at least one of the aforementioned nonparametric statistics exceeds their corresponding limit. As the authors documented, the resulting scheme has a balanced protection against different shift magnitudes, while the computational workload is fairly flimsy.

\subsection{A Nonparametric CUSUM Control Chart Based on the Lepage Statistic}

Chowdhury et al. (2015) introduced a single distribution-free CUSUM control chart for simultaneously monitoring the location and scale parameters of the underlying process. Their proposal is based on the utilization of the Lepage statistic, which is actually a combination of the well-known Wilcoxon rank sum statistics $T_{1}$ and the Ansari-Bradley test statistic $T_{2}$ (Lepage, 1971). Note that a nonparametric Shewhart-type monitoring scheme on the basis of the Lepage statistic has been also studied in the literature (Mukherjee and Chakraborti, 2012).

Let $X_{1}, X_{2}, \ldots, X_{m}$ be a group of magnitude $m$ collected randomly from the IC production. The $X$ observations form the so-called reference sample of the charting procedure. Afterwards, test samples of size $n Y_{j 1}, Y_{j 2}, \ldots, Y_{j n}, j=1,2, \ldots$ are drawn from the process in order to decide whether the process is still IC or a shift in either the location or the scale parameter has been occurred. Denoting by $\mu_{1}, \mu_{2}$ the mean values of random variables $T_{1}, T_{2}$ and by $\sigma_{1}, \sigma_{2}$ the corresponding standard deviations, the so-called Lepage statistic $S_{L j}^{2}, j=1,2, \ldots$ is computed for the $j$-th test sample, by the aid of the following formula

$S_{L j}^{2}=S_{1 j}^{2}+S_{2 j}^{2}$,

where

$S_{1 j}^{2}=\frac{T_{1}-\mu_{1}}{\sigma_{1}}, S_{2 j}^{2}=\frac{T_{2}-\mu_{2}}{\sigma_{2}},, j=1,2, \ldots$

Chowdhury et al. (2015) constructed their upper one-sided nonparametric control chart by monitoring the following CUSUM-type statistic

$C_{j}=\max \left(0, C_{j-1}+\left(S_{L j}^{2}-2\right)-k\right)$, 
where the parameter $k$ is the reference value, while the starting value $C_{0}$ equals to 0 .

It is of some interest to cast a glance at the follow-up study of the proposed chart. Indeed, Chowdhury et al. (2015) computed the $p$-values for the Wilcoxon rank sum test for location and the Ansari-Bradley test for scale respectively, on the basis of the two samples: one consisting of $m$ reference observations and the other with the $n$ observations from the $j$-th test sample. If $p_{1}$ and $p_{2}$ correspond to the $p$-values of the nonparametric tests applied for tracking down possible shifts in location and scale parameter respectively, then it is evident that if $p_{1}$ is very low but $p_{2}$ is not a shift in only location is indicated. On the other hand, if $p_{1}$ is relatively high but $p_{2}$ seems to be low then a shift only in scale is suspected. It is readily deduced that once both $p$-values are very low, a shift in both location and scale is declared. Note that a similar argumentation has been followed by Chowdhury et al. (2014), McCracken et al. (2013).

Chowdhury et al. (2015) investigated the performance of their proposed CUSUM scheme by taking into account different distributional models. For instance, their experimentation included the narrow-tailed Normal distribution, the heavy-tailed symmetric Cauchy distribution and the asymmetric Lognormal distribution. The supremacy of the Lepage technique over the rank-sum one is rationalized since one of its components is the rank-sum statistic. Consequently, any shift that the rank-sum statistic is able to track over, is also possible to be tracked over by the Lepage method.

Among other practical guidelines, Chowdhury et al. (2015) proposed to utilize smaller values of $k$ for tracking down slight to moderate location or scale changes under narrow-tailed and asymmetric distributions and slighter to bigger ones ubder heavy-tailed distributions.

\subsection{A Nonparametric CUSUM Control Chart Based on the Cucconi Statistic}

Mukherjee and Marozzi (2017) established a distribution-free CUSUM control chart which relies on the Cucconi statistic for concurrently detecting possible changes in the underlying distribution. It is evident that the joint monitoring for location and scale shifts has attracted a lot of research interest in the last two decades (Chinna et al., 2000; Cheng and Thaga, 2006).

Mukherjee and Marozzi (2017) considered the utilization of the so-called Cucconi test statistic, which has been first introduced by Cucconi (1968). As Marozzi $(2009,2013)$ argued, the Cucconi testing procedure seems to outperform the Lepage test for several cases (both light or heavy tailed, symmetric or asymmetric ones).

Let $X_{1}, X_{2}, \ldots, X_{m}$ be a group of magnitude $m$ drawn randomly from the IC production. The $X$ observations form the so-called reference sample of the charting procedure. Afterwards, several test samples of size $n Y_{j 1}, Y_{j 2}, \ldots, Y_{j n}, j=1,2, \ldots$ are drawn from the process in order to decide whether the process is still IC or a shift in either the location or the scale parameter has been occurred. The well-known Wilcoxon rank sum statistic $T_{1}$ for investigating the comparability between two location parameters, corresponds to the sum of the ranks of $Y$ 's in the combined group of size $m+n$ and it can be computed via the following formula

$T_{1}=\sum_{k=m+1}^{m+n} k I_{k}$ 
while its IC mean and variance are given by

$$
E\left(T_{1}\right)=\frac{n(m+n)}{2}, V\left(T_{1}\right)=\frac{m n(m+n+1)}{12}
$$

Note that function $I_{k}$ is simply an indicator variable, which corresponds to $1(0)$ if the $k$-th order statistic of the joint sample is a $X-(Y-)$ observation. Cucconi (1968) considered the sum $S_{1}$ of the squares of the ranks of $Y^{\prime} s$ in the combined sample, namely $S_{1}=\sum_{k=m+1}^{m+n} k^{2} I_{k}$.

Denoting by $T_{2}$ and $S_{2}$ the sum of the anti-ranks of $Y^{\prime}$ 's and the overall quantity of the squares of anti-ranks of $Y$ 's in the pooled group correspondingly, the following formulae hold true

$$
T_{2}=\sum_{k=m+1}^{m+n}(m+n+1-k) I_{k}=n(m+n+1)-T_{1}
$$

and

$$
S_{2}=\sum_{k=m+1}^{m+n}(m+n+1-k)^{2} I_{k}=n(m+n+1)^{2}-(m+n+1) T_{1}+S_{1} .
$$

If $W$ and $Z$ denote the standardized statistics of $S_{1}$ and $S_{2}$ defined previously, the Cucconi test statistic could now be readily delivered by the aid of the next formula

$C=\frac{W^{2}+Z^{2}-2 \rho X Y}{2(1-\rho)^{2}}$,

where $\rho$ corresponds to the correlation coefficient between $W$ and $Z$. For more details about the statistical framework of the so-called Cucconi testing procedure, the interested reader is referred to Marozzi (2009, 2013, 2014).

Mukherjee and Marozzi (2017) constructed their upper one-sided nonparametric control chart by monitoring the following CUSUM-type statistic

$$
C C_{j}=\max \left(0, C C_{j-1}+\left(C_{j}-1\right)-k\right), j=1,2, \ldots,
$$

where the parameter $k$ is the non-negative reference value, while the starting value $C C_{0}$ equals to 0 . The proposed CUSUM nonparametric control chart produces an OOC signal whenever $C C_{j}>H$, where $H>0$.

Following a parallel argumentation that the one proposed by Chowdhury et al. (2015), Mukherjee and Marozzi (2017) provided a follow-up study for investigating whether the observed shift refers to the location or scale parameter (or even both). 
According to the numerical results provided by Mukherjee and Marozzi (2017), their CUSUM scheme seems to perform better than the Lepage-type CUSUM one (see Subsection 4.4 of the present manuscript) in many situations. An interesting application of their control scheme addresses service quality monitoring by analyzing a quite realistic issue being associated with the Vancouver City (Canada) call center data.

\subsection{A Nonparametric CUSUM Control Chart Based on the Mann-Whitney Statistic}

Wang et al. (2017) established a monitoring scheme for tracking over the slight changes in the process mean, when the nominal value is unascertained but some past data are within reach. Their proposed monitoring scheme relies on the well-known Mann-Whitney test Mann and Whitney (1947), while a change-point model is also implemented. It is worth mentioning that the Mann Whitney statistic and its modifications have been already utilized for constructing Shewhart-type monitoring schemes Chakraborti and van de Wiel (2008).

Wang et al. (2017) considered a change-point model based on sequence of finite independent random quantities $\left\{X_{i}\right\}$, where $i=1,2, \ldots, l$. In fact, the change-point model utilized in their work is the common one defined in Eq. (43) of the present manuscript. The proposed CUSUM-type monitoring statistic $S_{j}$ calls for the determination of the Mann-Whitney statistic, which is defined for $1 \leq t<l$ as follows

$$
M W_{t, l}=\sum_{i=1}^{t} \sum_{j=t+1}^{l} I\left(x_{j}<x_{i}\right)=\sum_{j=t+1}^{l}\left(I\left(x_{j}<x_{1}\right)+I\left(x_{j}<x_{2}\right)+\ldots+I\left(x_{j}<x_{t}\right)\right),
$$

where

$$
I\left(x_{j}<x_{i}\right)=\left\{\begin{array}{l}
1, x_{j}<x_{i} \\
0, x_{j} \geq x_{i}
\end{array}\right.
$$

Under the assumption that no shift has been occurred, the mean and variance of the test statistic defined in Eq. (58) are given by

$$
E\left(M W_{t, l}\right)=\frac{t(l-t)}{2}, V\left(M W_{t, l}\right)=\frac{t(l-t)(l+1)}{12}
$$

Based on the above-mentioned argumentation, one may readily obtain the standardized Mann Whitney statistic $S M W_{t, l}$ and consequently reach the CUSUM-type monitoring statistic proposed by Wang et al. (2017), which is given as

$S_{j}^{+}(a, b)=\max \left(0, S_{j-1}^{+}(a, b)+S M W_{j,(a+b)}-k\right)$,

$S_{j}^{-}(a, b)=\max \left(0, S_{j-1}^{-}(a, b)+S M W_{j,(a+b)}+k\right)$,

where $j=a-a_{0}, a-a_{0}+1, \ldots, a-a_{0}+b-1,0 \leq a_{0} \leq a$, while the starting values for both monitoring statistics are equal to zero. Throughout the lines of the work of Wang et al. (2017), the reference parameter $k$ is assumed to be equal to 0.5. Their proposed CUSUM nonparametric control chart produces an OOC signal if at least one of the following is satisfied 
$S_{\max }^{+}(a, b) \leq h_{a, b}$ or $S_{\min }^{-}(a, b) \geq-h_{a, b}$,

where the decision value $h_{a, b}$ is appropriately determined such that a pre-specified level of performance is achieved, while

$S_{\max }^{+}(a, b)=\max _{a-a_{0} \leq j \leq a+b-1} S_{j}^{+}(a, b), S_{\min }^{-}(a, b)=\max _{a-a_{0} \leq j \leq a+b-1} S_{j}^{-}(a, b)$.

An extensive numerical experimentation provided by Wang et al. (2017) revealed that their proposed CUSUM-type nonparametric control chart seems to be slightly more efficient versus other distribution-free monitoring schemes such as those established by Bakir and Reynolds (1979), Zhou et al. (2009), Yang and Cheng (2011).

\subsection{An Adaptive Distribution-Free CUSUM Scheme Based on Sequential Ranks}

Lang (2019) studied an adaptive self-starting distribution-free CUSUM control chart based on sequential ranks. An effective calibration algorithm for evaluating its performance is provided, while several ready-to-use numerical results concerning its corresponding control limits are also produced. The aim of the corresponding chart is to detect possible departures from the underlying sampling distribution to a stochastically larger distribution.

The idea behind the adaptive rank-based decision rule, is to alleviate the drawbacks of CUSUMtype schemes, while maintaining their ease-of-use and robustness. This purpose can be advanced by the work of Lang (2019), which has been relied on a distribution-free bootstrap based CUSUM control chart established by Chatterjee and Qiu (2009).

We next denote by $R_{t}$ the $t$-th sequential rank, namely the rank of observed value $x_{t}$ between the observations $x_{1}, x_{2}, \ldots, x_{t}$. which can be expressed as

$$
R_{t}=1+\sum_{j=1}^{t-1}\left(x_{t}-x_{j}\right)^{+}
$$

where $(x)^{+}$takes on the value 1 if $x>0$, while it equals zero otherwise. The CUSUM-type monitoring statistic utilized by Lang (2019) is given by

$$
C_{S R_{t}}=\max \left(0, C_{S R_{t-1}}+\frac{R_{t}}{t+1}-k\right), t \geq 1
$$

where $k$ corresponds to the reference value, while the respective starting values are equal to zero. Lang (2019) defined next the random variable $Y_{S R_{j}}$, which follows the conditional distribution given below

$$
Y_{S R_{j}} \sim\left(C_{S R_{t}} \mid T_{S R_{t}}=j\right)
$$


where the so-called sprint length $T_{S R_{t}}$ corresponds to the time elapsed since $C_{S R_{t}}$ was last zero and can be expressed as

$$
T_{S R_{t}}=\left\{\begin{array}{l}
0, \text { if } C_{S R_{t}}=0 \\
j, \text { if } C_{S R_{t}} \neq 0, \ldots, C_{S R_{t-j+1}} \neq 0 \text { and } C_{S R_{t-j}}=0 \text { for } j=1,2, \ldots, n
\end{array}\right.
$$

Since the conditional distribution of $Y_{S R_{j}}$ depends only on $j$ and $F$ and not on $n$ Chatterjee and Qiu (2009), the corresponding unconditional distribution of $C_{S R_{t}}$ can be readily derived as

$$
C_{S R_{t}} \sim \sum_{j=1}^{j_{\max }} Y_{S R_{j}} I\left(T_{S R_{t}}=j\right)+Y^{*} I\left(T_{S R_{t}}>j_{\max }\right), \text { for } j_{\max } \leq n
$$

where $I$ denotes the common indication variable, while $Y^{*} \sim\left(C_{S R_{t}} \mid T_{S R_{t}}>j_{\max }\right)$. The upper onesided adaptive CUSUM nonparametric monitoring scheme proposed by Lang (2019) gives an alarm whenever one of the following holds true

$$
T_{S R_{t}}=j \text { and } C_{S R_{t}}>h_{j}, 1 \leq j \leq j_{\max }
$$

or

$$
T_{S R_{t}}>j_{\max } \text { and } C_{S R_{t}}>h_{j_{\max }},
$$

where the control limits $h_{j}$ can be determined by the aid of an appropriate Monte-Carlo simulation procedure. As Chatterjee and Qiu (2009) recommended, the limits $h_{j}$ are computed up to a rationally low $j_{\max }$ while after that, if the monitoring statistic does not bounce back to 0 , then $h_{j}$ remain equal to $h_{j_{\max }}$.

Lang (2019) evaluated the performance of its proposal by the aid of several characteristics, such as the FAR, the ARL and the Detection Delay Time. Based on his numerical experimentation, the onesided adaptive CUSUM nonparametric control chart seems to outperform several distribution-free competitors.

\subsection{A Nonparametric CUSUM Control Chart Based on the Hogg-Fisher-Randle Statistic}

Mukherjee et al. (2019) introduced CUSUM-type schemes which rely on the so-called HoggFisher-Randles (HFR, hereafter) statistic in order to investigate slight and persistent changes in particular characteristics of the underlying process. Gastwirth (1965) increased the efficiency of rank-based testing procedures by offering some ranks of the combined samples. Following the above-mentioned framework, Hogg et al. (1975) established the so-called HFR statistic, which could be viewed as a different choice to the Wilcoxon rank-sum statistic defined earlier, for dealing with the two-sample location problem. Note that the above-mentioned HFR test statistic has been 
widely utilized (Thas et al., 2012; Mukherjee and Sen, 2015). In fact, as it can be easily observed, the HFR statistic is strongly related to the Wilcoxon one.

Let $X_{1}, X_{2}, \ldots, X_{m}$ be a group of magnitude $m$ drawn randomly from the IC distribution. The $X$ observations form the so-called reference sample of the charting procedure. Afterwards, test samples of magnitude $n \quad Y_{j 1}, Y_{j 2}, \ldots, Y_{j n}, j=1,2, \ldots$ are drawn for determining the status of the process. Denoting by $R_{i j}$ the rank of observations $Y_{i j}$ in the combined sample of size $(m+n)$, the $H F R$ statistic $T_{\mathrm{HFR}, i}$ is given by

$T_{H F R, i}=\sum_{j: R_{i j} \leq K} R_{i j}, \quad$ for $[(m+n+1) / 2] \leq K \leq m+n$,

where $[x]$ denotes the greatest integer contained in $x$. Since Hogg et al. (1975) thought of the sum of ranks of only those $Y$ groups, whose ranks are smaller than or equal to the median rank of the combined samples, the HFR statistic seems to be suitable for detecting possible shifts in location parameter in positively asymmetric distributions.

The IC mean and variance of $H F R$ test statistic are given by Hogg et al. (1975).

$$
E\left(T_{H F R}\right)=\frac{n K(K+1)}{2(m+n)}, V\left(T_{H F R}\right)=\frac{m n K(K+1)\left(-3 K^{2}+K(4(m+n)-3)+2(m+n)\right)}{12(m+n-1)(m+n)^{2}} .
$$

Based on the above-mentioned argumentation, one may readily obtain the standardized $H F R$ statistic $S H F R_{s, i}$ and consequently reach the CUSUM-type monitoring statistic proposed by Mukherjee et al. (2019), which is given as

$C_{s, i}=\max \left(0, C_{s, j-1}+\operatorname{SHFR}_{s, i}-k\right), i=1,2, \ldots$

where the starting value is equal to zero and $k$ corresponds to the reference parameter.

The upper one-sided CUSUM nonparametric control chart established by Mukherjee et al. (2019) produces an OOC signal if $C_{s, i}>H$, where $H>0$ denotes the upper control limit. Note that a simple modification for covering possible two-sided shifts is also proposed. More precisely, Mukherjee et al. (2019) mentioned that one could readily modulate the control schemes described above for twosided shifts by utilizing the absolute value of the corresponding HFR test statistic.

A thorough numerical experimentation brought off by the same authors, included the utilization of several distributions, such as the Normal distribution, the Exponential distribution, the Gamma distribution, the Log-Logistic distribution, the Laplace distribution and the Log-normal distribution. Based on their results, a variety of interesting comments and instructive remarks are stated in order to shed light on the IC and OOC behavior of the corresponding CUSUM scheme. It is numerically verified that the new scheme outperforms other traditional nonparametric charts in most of the cases considered, especially for small and moderate shifts of the underlying the rightskewed distributions. Generally speaking, the proposed control chart seems to be quite capable for monitoring an industrial process under right-skewed distributional models. 
Mukherjee et al. (2019) illustrated their proposed nonparametric CUSUM charts by utilizing real data from iron ore mining plant. The quality measurement therein expresses the percentage of silica concentrate that remains as an impurity at the end of the froth flotation process. The outcomes were really encouraging for their CUSUM chart.

\subsection{A Mixed GWMA-CUSUM Control Chart Based on the Mann Whitney Statistic}

Mabude et al. (2020) established capable distribution-free charts, known as mixed generally weighted moving average-cumulative sum Mann Whitney (GWMA-CUSUM-MW, hereafter) chart as well as its reversed edition, i.e. the CUSUM-GWMA Mann Whitney chart (CUSUM-GWMA$M W$, hereafter). The authors investigated the behavior of the corresponding monitoring schemes by the aid of their corresponding $A R L$ and $A E Q L$.

In an effort to increase the ability of the so-called parametric GWMA and CUSUM schemes in detecting small shifts, Lu (2017) proposed the mixed GWMA-CUSUM scheme and its reversed edition, i.e. CUSUM-GWMA chart for individual observations. Ali and Haq (2018), Lu (2017) were firstly established GWMA-CUSUM monitoring schemes for monitoring random samples of size equal to or larger than 1.

Let $X_{1}, X_{2}, \ldots, X_{m}$ be a group of magnitude $m$ collected randomly from the IC production. The $X$ observations form the so-called reference sample of the charting procedure. Afterwards, test samples of size $n Y_{j 1}, Y_{j 2}, \ldots, Y_{j n}, j=1,2, \ldots$ are drawn for deciding about the status of the process. Recalling the Mann-Whitney statistic Mann and Whitney (1947), it is readily observed that, under the assumption that there are no ties, its mean is $\mu_{U}$ equal to $m n / 2$. On the other hand, Mabude et al. (2020) followed the approach established by Sheu and Lin (2003) and defined the monitoring statistic $G_{t}$ of their GWMA chart as

$$
G_{t}=\sum_{i=1}^{t}\left(q^{(i-1) a}-q^{i^{a}}\right) U_{t-i+1}+q^{t^{a}} U_{0}, t=1,2,3, \ldots
$$

where $q \in[0,1)$ and $a>0$ are two design parameters which are appropriately determined.

The proposed GWMA-CUSUM-MW chart can now be constructed by combining the GWMA chart and the CUSUM chart, which utilize the Mann-Whitney test statistic. Consequently, the plotted statistic of the nonparametric scheme established by Mabude et al. (2020) are given by

$$
M G C_{t}^{+}=\max \left(0, M G C_{t-1}^{+}+G_{t}-K_{t}-\mu_{U}\right), t=1,2, \ldots
$$

and

$$
M G C_{t}^{-}=\max \left(0, M G C_{t-1}^{-}+\mu_{U}-G_{t}-K_{t}\right), t=1,2, \ldots
$$

where the starting values are equal to zero and the time-varying reference value $K_{t}$ can be expressed by the aid of the corresponding reference parameter $k$ as 
$K_{t}=k \sqrt{V\left(G_{t}\right)}$,

where $V\left(G_{t}\right)$ corresponds to the variance of the random variable $G_{t}$. It is straightforward that the proposed upper one-sided nonparametric control chart gives an alarm whenever at least one of the monitoring statistics falls on or above the upper control limit for any value of $t$. Mabude et al. (2020) followed a parallel argumentation for constructing also the so-called CUSUM-GWMA-MW control chart. Some recent advances on mixed monitoring schemes, the interested researcher is referred to Abid et al. (2021).

\subsection{A Nonparametric CUSUM Control Chart Based on a Modified Lepage-Type Statistic}

Tercero-Gomez et al. (2020) established a CUSUM-type control chart based on a Lepage-type statistic that combines the Wilcoxon rank sum statistic and the Mood statistic Hajek et al. (2019), Mood (1954). The corresponding control limits and the IC and OOC performance of the respective CUSUM-type chart are evaluated by the aid of a Monte-Carlo simulation procedure.

Tercero-Gomez et al. (2020) defined a Lepage-type monitoring statistic in the sense that they considered the sum of two distribution-free test statistics (the first one for location and the other for scale parameter). As expected, their Lepage-type statistic $L_{M}$ is proved to be sensitive not only to mean shifts but also to variances changes.

Let $X_{1}, X_{2}, \ldots, X_{m}$ denote a group of magnitude $m$ collected randomly from the IC production. The $X$-observations form the so-called reference sample of the charting procedure. Afterwards, several test samples of size $n Y_{j 1}, Y_{j 2}, \ldots, Y_{j n}, j=1,2, \ldots$ are drawn from the process in order to decide whether the process is still IC or not. The statistic $L_{M}$ is defined by the aid of the Wilcoxon rank sum statistic $W$ and the Mood Statistic $M$ as follows

$$
L_{M}=W^{2}+M^{2}
$$

Consequently, the CUSUM-type statistic of the nonparametric control chart established by TerceroGomez et al. (2020) is given by

$$
C_{j}=\max \left(0, C_{j-1}+L_{M}-2-k\right), j>0,
$$

where the starting value is equal to zero and the parameter $k$ corresponds to the reference value. The proposed upper one-sided nonparametric scheme gives an alarm every time that $C_{j}$ falls on or above the upper control limit for any value of $j$.

Based on the numerical results provided by Tercero-Gomez et al. (2020), their proposed CUSUMtype nonparametric control chart outperforms the corresponding one established by Chowdhury et al. (2015).

\section{Conclusions}

Nonparametric CUSUM control charts do not demand the presumption of any specific probability distribution for the underlying process. In addition, distribution-free control charting is likely to 
possess the robustness feature of standard nonparametric procedures and consequently there exists less possibility to be influenced by outliers, or the presence of asymmetric or heavy-tailed distributions for the underlying populations. It is evident that the research activity on the topic of nonparametric CUSUM schemes is intensively activated and it seems to be strengthened due to their consistent theoretical framework and their appealing practical implementation. Throughout the lines of the present manuscript, a thorough and up-to-date review of relative advances has been taken place, while approximately 100 published articles and books in related fields are cited. It is evident that CUSUM nonparametric control charts have brought near a lot of research attention in the last decade. Their simplicity and ability to monitor effectively an underlying process even if its distribution is unknown or non-normal, turns them to a very useful statistical tool for improving the quality of a product. However, the topic seems to dispose more space for further scientific research. The implementation of additional nonparametric test statistics or their combination with traditional or more purpose-built change-point models could give birth to new CUSUM monitoring schemes. Some attractive applications of CUSUM control charts in health care and monitoring, which have appeared recently in the literature Keshavarz et al. (2021), seems to provide new perspectives on the topic. Moreover, the utilization of bootstrap methods, which have been already proved that they provide an alternative but reliable way for constructing CUSUM schemes, could be expanded further and may result in interesting generalizations of the existing charting procedures. Finally, some effort must be done for constructing CUSUM schemes for joint monitoring possible changes in location and scale parameters of the underlying distribution process.

\section{Conflicts of Interest}

The authors declare no conflict of interest.

\section{Acknowledgements}

The authors thank two anonymous referees for several helpful comments and suggestions on an earlier version of the manuscript, which resulted in some improvement of the present article.

\section{References}

Abid, M., Mei, S., Nazir, H.Z., Riaz, M., \& Hussain, S. (2021). A mixed HWMA-CUSUM mean chart with an application to manufacturing process. Quality and Reliability Engineering International, 37(2), 618631.

Abid, M., Nazir, H.Z., Riaz, M., \& Lin, Z. (2017). Investigating the impact of ranked set sampling in nonparametric CUSUM control charts. Quality and Reliability Engineering International, 33(1), 203214.

Abid, M., Nazir, H.Z., Tahir, M., \& Riaz, M. (2018). On designing a new cumulative sum, Wilcoxon signed rank chart for monitoring process location, PloS One, 13(4), 1-18.

Abujiya, M., \& Muttlak, H. (2004). Quality control chart for the mean using double ranked set sampling. Journal of Applied Statistics, 31(10), 1185-1201.

Ali, R., \& Haq, A. (2018). A mixed GWMA-CUSUM control chart for monitoring the process mean. Communications in Statistics-Theory and Methods, 47(15), 3779-3801.

Amin, R.W., Reynolds Jr., M.R., \& Saad, B. (1995). Nonparametric control charts based on sign statistics. Communication in Statistics-Theory \& Methods, 24(6), 1597-1623.

Asghari, S., Gildeh, B.S., Ahmadi, J., \& Borzadaran, G.M. (2018). Sign control chart based on ranked set sampling. Quality Technology \& Quantitative Management, 15(5), 568-588. 
Bagshaw, M., \& Johnson, R.A. (1975). The influence of reference values and estimated variance on the ARL of CUSUM tests. Journal of the Royal Statistical Society: Series B (Methodological), 37(3), 413-420.

Bakir, S.T., \& Reynolds, M.R. (1979). A nonparametric procedure for process control based on within-group ranking. Technometrics, 21(2), 175-183.

Balakrishnan, N., \& Ng, H.K.T. (2006). Precedence-type tests and applications (Vol. 472). John Wiley \& Sons, New York.

Balakrishnan, N., Triantafyllou, I.S., \& Koutras, M.V. (2009). Nonparametric control charts based on runs and Wilcoxon-type rank-sum statistics. Journal of Statistical Planning and Inference, 139(9), 3177-3192.

Bohn, L.L., \& Wolfe, D.A. (1992). Nonparametric two-sample procedures for ranked set samples data. Journal of American Statistical Association, 87(418), 522-561.

Bohn, L.L., \& Wolfe, D.A. (1994). The effect of imperfect judgment rankings on properties of procedures based on the ranked-set samples analog of the Mann-Whitney-Wilcoxon statistic. Journal of the American Statistical Association, 89(425), 168-176.

Brook, D., \& Evans, D.A. (1972). An approach to the probability distribution of CUSUM run length. Biometrika, 59(3), 539-549.

Brown, A.R. (2020). The alternative distribution of the nonparametric extended median test CUSUM chart for multiple stream processes. Communication in Statistics-Theory and Methods, 1-11. DOI: 10.1080/03610926.2020.1850792.

Brown, A.R., \& Schaffer, J.R. (2020). A nonparametric CUSUM control chart for multiple stream processes based on a modified extended median test. Communications in Statistics-Theory and Methods, 1-14. DOI: 10.1080/ 03610926.2020.1738492.

Castagliola P., Tran K.P., Celano G., \& Maravelakis P.E. (2020). The Shewhart sign chart with ties: performance and alternatives. In: Koutras, M.V., Triantafyllou, I.S. (eds) Distribution-Free Methods for Statistical Process Monitoring and Control. Springer, Berlin, pp. 107-136.

Chakraborti S., \& Graham, M. (2019a). Nonparametric statistical process control. John Wiley \& Sons, U.S.A.

Chakraborti, S., \& Graham, M.A. (2019b). Nonparametric (distribution-free) control charts: an updated overview and some results. Quality Engineering, 31(4), 523-544.

Chakraborti, S., \& Van de Wiel, M.A. (2008). A nonparametric control chart based on the mann-whitney statistic. In: Balakrishnan, N., Peña, E.A., Silvapulle, M.J. (eds) Beyond Parametrics in Interdisciplinary Research: Festschrift in honor of Professor Pranab. K. Sen, IMS Collections. Beachwood, Ohio, USA, vol. 1, pp. 156-172.

Chakraborti, S., Van der Laan, P., \& Bakir, S.T. (2001). An overview and some results. Journal of Quality Technology, 33(3), 304-315.

Chatterjee, S., \& Qiu, P. (2009). Distribution-free cumulative sum control charts using bootstrap-based control limits. The Annals of Applied Statistics, 3(1), 349-369.

Cheng, S.W., \& Thaga, K. (2006). Single variables control charts: an overview. Quality and Reliability Engineering International, 22(7), 811-820.

Chinna, K., Kadir, S.L.S.A., \& Abdullah, M. (2000). Box-chart: combining x and S charts. Total Quality Management, 11(4-6), 857-862.

Chowdhury, S., Mukherjee, A., \& Chakraborti, S. (2014). A new distribution-free control chart for joint monitoring of location and scale parameters of continuous distributions. Quality and Reliability Engineering International, 30(2), 191-204. 
Chowdhury, S., Mukherjee, A., \& Chakraborti, S. (2015). Distribution free phase II CUSUM control chart for joint monitoring of location and scale. Quality and Reliability Engineering International, 31(1), 135151.

Conover, W.J. (1998). Practical nonparametric statistics. John Wiley \& Sons, New York.

Cucconi, O. (1968). Un nuovo test non parametrico per il confronto fra due gruppi di valori campionari. Giornale degli Economisti e Annali di Economia, 27(3-4), 225-248.

Frey, J., \& Zhang, Y. (2021). Robust confidence intervals for a proportion using ranked-set sampling. Journal of the Korean Statistical Society. DOI: 10.1007/s42952-020-00103-3.

Gan, F.F. (1993). An optimal design of CUSUM control charts for binomial counts. Journal of Applied Statistics, 20(4), 445-460.

Gastwirth, J.L. (1965). Percentile modifications of two-sample rank tests. Journal of the American Statistical Association, 60(312), 1127-1141.

Graham, M.A., Chakraborti, S., \& Mukherjee, A. (2014). Design and implementation of CUSUM exceedance control charts for unknown location. International Journal of Production Research, 52(18), 5546-5564.

Hajek, J., Sidak, Z., \& Sen, P. (2019). Theory of rank tests. Elsevier, San Diego, California, U.S.A.

Haq, A., Brown, J., \& Moltchanova, E. (2015). New synthetic control charts for monitoring process mean and process dispersion. Quality and Reliability Engineering International, 31(8), 1305-1325.

Hawkins, D.M. (1992). A fast approximation for average run length of CUSUM control charts. Journal of Quality Technology, 24(1), 37-43.

Hawkins, D.M., \& Deng, Q. (2010). A nonparametric change point control chart. Journal of Quality Technology, 42(2), 165-173.

Hettmansperger, T.P. (1995). The ranked-set sample sign test. Journal of Nonparametric Statistics, 4(3), 263-270.

Hogg, R.V., Fisher, D.M., \& Randles, R.H. (1975). A two-sample adaptive distribution-free test. Journal of American Statistical Association, 70(351a), 656-661.

Janacek, G.J., \& Meikle, S.E. (1997). Control charts based on medians. Journal of the Royal Statistical Society: Series D (The Statistician), 46(1), 19-31.

Keshavarz, M., Asadzadeh, S., \& Niaki, S.T.A. (2021). Risk-adjusted frailty-based CUSUM control chart for phase I monitoring of patients' lifetime. Journal of Statistical Computation and Simulation, 91(2), 334-352.

Khoo, M.B.C., Wong, V.H., Wu, Z., \& Castagliola, P. (2011). Optimal designs of the multivariate synthetic chart for monitoring the process mean vector based on the median run length. Quality and Reliability Engineering International, 27(8), 981-997.

Kim, D.H., \& Kim, Y.C. (1996). Wilcoxon signed rank test using ranked-set sample. Korean Journal of Computational \& Applied Mathematics, 3(2), 235-243.

Koshti, R.D., \& Kamalja, K.K. (2021). Parameter estimation of Cambanis-type bivariate uniform distribution with ranked set sampling. Journal of Applied Statistics, 48(1), 61-83.

Koutras, M.V., \& Triantafyllou, I.S. (2020). Recent advances on univariate Shewhart type distribution-free Shewhart-type control charts. In: Koutras, M.V., Triantafyllou, I.S. (eds) Distribution-free Methods for Statistical Process Monitoring and Control. Springer, Berlin, pp. 1-56.

Lang, M. (2019). Control limits for an adaptive self-starting distribution-free CUSUM based on sequential ranks. Technologies, 7(4), 71-89. 
Lepage, Y. (1971). A combination of Wilcoxon's and Ansari-Bradley's statistics. Biometrika, 58(1), 213217.

Li, S.Y., Tang, L.C., \& Ng, S.H. (2010). Nonparametric CUSUM and EWMA control charts for detecting mean shifts. Journal of Quality Technology, 42(2), 209-226.

Liu, L., Tsung, F., \& Zhang, J. (2014). Adaptive nonparametric CUSUM scheme for detecting unknown shifts in location. International Journal of Production Research, 52(6), 1592-1606.

Liu, L., Zhang, J., \& Zi, X. (2015). Dual nonparametric CUSUM control chart based on ranks. Communications in Statistics-Simulation and Computation, 44(3), 756-772.

Lu, S.L. (2017). Novel design of composite generally weighted moving average and cumulative sum charts. Quality and Reliability Engineering International, 33(8), 2397-2408.

Lucas, J.M., \& Saccucci, M.S. (1990). Exponentially weighted moving average control schemes: properties and enhancements. Technometrics, 32(1), 1-12.

Mabude, K., Malela-Majika, J.C., Castagliola, P., \& Shongwe, S.C. (2020). Distribution-free mixed GWMACUSUM and CUSUM-GWMA Mann-Whitney charts to monitor unknown shifts in the process location. Communication in Statistics-Simulation and Computation, 1-24. DOI: 10.1080/03610918.2020.1811331.

Mann, H.B., \& Whitney, D.R. (1947). On a test whether one of two random variables is stochastically larger than other. The Annals of Mathematical Statistics, 18(1), 50-60.

Manoukian, E.B. (1986). Mathematical nonparametric statistics. Gordon and Breach Science Publishers, S.A.

Marozzi, M. (2009). Some notes on the location-scale Cucconi test. Journal of Nonparametric Statistics, 21(5), 629-647.

Marozzi, M. (2013). Nonparametric simultaneous tests for location and scale testing: a comparison of several methods. Communications in Statistics-Simulation and Computation, 42(6), 1298-1317.

Marozzi, M. (2014). The multisample Cucconi test. Statistical Methods and Applications, 23(2), 209-227.

McCracken, A.K., Chakraborti, S., \& Mukherjee, A. (2013). Control charts for simultaneous monitoring of unknown mean and variance of normally distributed processes. Journal of Quality Technology, 45(4), 360-376.

McDonald, D. (1990). A CUSUM procedure based on sequential ranks. Naval Research Logistics, 37(5), 627-646.

McGilchrist, C.A., \& Woodyer, K.D. (1975). Note on a distribution-free CUSUM technique. Technometrics, 17(3), 321-325.

McIntyre, G.A. (1952). A method for unbiased selective sampling using ranked sets. Australian Journal of Agricultural Research, 3(4), 385-390.

Mehmood, R., Riaz, M., \& Does, R.J.M.M. (2013). Control charts for location based on different sampling schemes. Journal of Applied Statistics, 40(3), 483-494.

Meneces, N.S., Olivera, S.A., Saccone, C.D., \& Tessore, J. (2008). Statistical control of multiple-stream processes: a Shewhart control chart for each stream. Quality Engineering, 20(2), 185-194.

Montgomery, D.C. (2007). Introduction to statistical quality control. John Wiley \& Sons, New York.

Mood, A.M. (1954). On the asymptotic efficiency of certain nonparametric two-sample tests. The Annals of Mathematical Statistics, 25(3), 514-522.

Mukherjee A., \& Chakraborti, S. (2012). A distribution-free control chart for joint monitoring of location and scale. Quality and Reliability Engineering International, 28(3), 335-352. 
Mukherjee, A., \& Marozzi, M. (2017). A distribution-free phase-II CUSUM procedure for monitoring service quality. Total Quality Management \& Business Excellence, 28(11-12), 1227-1263.

Mukherjee, A., \& Sen, R. (2015). Comparisons of Shewhart-type rank based control charts for monitoring location parameters of univariate processes. International Journal of Production Research, 53(14), 44144445.

Mukherjee, A., Chong, Z.L., \& Khoo, M.B.C. (2019). Comparisons of some distribution-free CUSUM and EWMA schemes and their applications in monitoring impurity in mining process flotation. Computers \& Industrial Engineering, 137, 106059.

Mukherjee, A., Graham, M.A., \& Chakraborti, S. (2013). Distribution free exceedance CUSUM control charts for location. Communications in Statistics-Simulation and Computation, 42(5), 1153-1187.

Nelson, L.S. (1963). Tables for a precedence life tests. Technometrics, 5(4), 491-499.

Page, E.S. (1954). Continuous inspection schemes. Biometrika, 4l(1/2), 100-115.

Perdikis, T., Psarakis, S., Castagliola, P., \& Maravelakis, P. (2021). An EWMA signed ranks control chart with reliable run length performances. Quality and Reliability Engineering International, 37(3), 12661284. DOI: $10.1002 /$ qre.2795.

Qiu, P. (2008). Distribution-free multivariate process control based on log-linear modeling. IIE Transactions, 40(7), 664-677.

Qiu, P. (2014). Introduction to statistical process control. CRC Press, Taylor \& Francis Group, New York.

Qiu, P. (2018). Some perspectives on nonparametric statistical process control. Journal of Quality Technology, 50(1), 49-65.

Qiu, P., \& Hawkins, D. (2001). A rank-based multivariate CUSUM procedure. Technometrics, 43(2), 120132.

Qiu, P., \& Li, Z. (2011a). On nonparametric statistical process control of univariate processes. Technometrics, 53(4), 390-405.

Qiu, P., \& Li, Z. (2011b). Distribution-free monitoring of univariate processes. Statistics \& Probability Letters, 81(12), 1833-1840.

Reynolds, M.R. (1975). Approximations to the average run length in cumulative control charts. Technometrics, 17(1), 65-71.

Runger, G.C., Alt, F.B., \& Montgomery, D.C. (1996). Controlling multiple stream processes with principal components. International Journal of Production Research, 34(11), 2991-2999.

Sheu, S.H., \& Lin, T.C. (2003). The generally weighted moving average control chart for detecting small shifts in the process mean. Quality Engineering, 16(2), 209-231.

Shirke, D.T., \& Barale, M.S. (2018). A nonparametric CUSUM chart for process dispersion. Quality and Reliability Engineering International, 34(5), 858-866.

Tercero-Gomez, V., Aguilar-Lleyda, V., Cordero-Franco, A., \& Conover, W. (2020). A distribution-free CUSUM chart for joint monitoring of location and scale based on the combination of Wilcoxon and Mood statistics. Quality and Reliability Engineering International, 36(4), 1422-1453.

Thas, O., Clement, L., Rayner, J.C.W., Carvalho, B., \& Van Criekinge, W. (2012). An omnibus consistent adaptive percentile modified Wilcoxon rank sumtest with applications in gene expression studies. Biometrics, 68(2), 446-454.

Triantafyllou, I.S. (2018). Nonparametric control charts based on order statistics: some advances. Communication in Statistics-Simulation and Computation, 47(9), 2684-2702. 
Triantafyllou, I.S. (2019). A new distribution-free control scheme based on order statistics. Journal of Nonparametric Statistics, 31(1), 1-30.

Triantafyllou, I.S. (2021). Wilcoxon-type rank sum control charts based on progressively censored reference data. Communication in Statistics-Theory and Methods, 50(2), 311-328.

Triantafyllou, I.S., \& Panayiotou, N. (2020). A new distribution-free monitoring scheme based on ranks, Communication in Statistics-Simulation and Computation, 1-23. DOI: 10.1080/ 03610918.2020.1804584.

Van Dobben de Bruyn, C.S. (1968). Cumulative sum tests: theory and practice. Griffin, London.

Wang, D., Zhang, L., \& Xiong, Q. (2017). A nonparametric CUSUM control chart based on the MannWhitney statistic. Communications in Statistics-Theory and Methods, 46(10), 4713-4725.

Wilcoxon, F. (1945). Individual comparisons by ranking methods. Biometrics Bulletin, 1(6), 80-83.

Woodall, W.H. (2000). Controversies and contradictions in statistical process control. Journal of Quality Technology, 32(4), 341-350.

Woodall, W.H., \& Montgomery, D.C. (1999). Research issues and ideas in statistical process control. Journal of Quality Technology, 31(4), 376-386.

Xue, L., \& Qiu, P. (2020). A nonparametric CUSUM chart for monitoring multivariate serially correlated processes. Journal of Quality Technology, 1-14. DOI: 10.1080/00224065.2020. 1778430.

Yang, S.F., \& Cheng, W.S. (2011). A new non-parametric CUSUM sign control chart. Quality and Reliability Engineering International, 27(7), 867-875.

You, L., \& Qiu, P. (2020). A nonparametric control chart for dynamic disease risk monitoring. In: Koutras, M.V., \& Triantafyllou, I.S. (eds) Distribution-free Methods for Statistical Process Monitoring and Control. Springer, Berlin, pp. 243-257.

Zhou, C., Zou, C., Zhang, Y., \& Wang, Z. (2009). Nonparametric control chart based on change-point model. Statistical Papers, 50(1), 13-28. 